\title{
Analysis of extended haplotype in Korean cattle (Hanwoo) population
}

\author{
Dajeong Lim ${ }^{1}$, Bong Hwan Choi ${ }^{1}$, Yong Min Cho ${ }^{1}$, Han Ha Chai , Gul Won Jang ${ }^{1}$, Cedric Gondro ${ }^{2}$, \\ Yeoung Ho Jeoung ${ }^{3}$ E Seung Hwan Lee, ${ }^{4}$, \\ ${ }^{1}$ Division of Animal Genomics \& Bioinformatics, National Institute of Animal Science, RDA, Jeonju 55365, Korea, ${ }^{2}$ School of \\ Environmental and Rural Science, University of New England, Armidale, NSW 2351, Australia, ${ }^{3}$ Hanwoo Department, Korea Animal \\ Improvement Association, Seoul 06668, ${ }^{4}$ Division of Animal and Dairy Science, Chung Nam National University, Daejeon 34134, Korea
}

\begin{abstract}
Korean cattle (Hanwoo) are categorized into three breeds based on color: brown, brindle, and black. Among these breeds, brown Hanwoo has been subjected to intensive selection to improve meat traits. To identify genetic traces driven by recent selection in brown Hanwoo, we scanned the genomes of brown and brindle Hanwoo using a bovine SNP chip. We identified 17 candidate selection signatures in brown Hanwoo and sequenced four candidate regions from 10 individuals each of brown and brindle Hanwoo. In particular, non-synonymous SNPs in the ADSL gene $(\mathrm{K88M}, \mathrm{L189H}$, and R302Q) might have had mutational effects on protein structure as a result of altering the purine pathway during nucleotide breakdown. The ADSL gene was previously reported to affect meat quality and yield in livestock. Meat quality and yield are main breeding goals for brown Hanwoo, and our results support a potential causal influence of non-synonymous SNPs in the ADSL gene. [BMB Reports 2016; 49(9): 514-519]
\end{abstract}

\section{INTRODUCTION}

Korean cattle exhibit three different color phenotypes: brown, brindle, and black (1). Brindle and black Hanwoo are the most closely related within breeds and among Hanwoo populations based on microsatellite markers analysis. However, brindle and black Hanwoo still have particular genetic characteristics that were highly significantly different $(P<0.001)$ based on

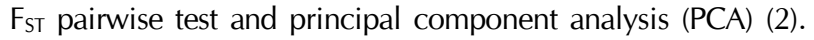
In particular, brown Hanwoo has become highly specialized for meat traits after undergoing strong artificial selection (3). Since the Hanwoo breeding program was started in the 1930s,

${ }^{*}$ Corresponding author. Tel: +82-42-821-5772; Fax: +82-42-8259754; E-mail: slee46@cnu.ac.kr

http://dx.doi.org/10.5483/BMBRep.2016.49.9.074

Received 25 April 2016, Revised 30 May 2016, Accepted 27 July 2016

Keywords: ADSL, Brindle Hanwoo, Extendend haplotype, Hanwoo brown Hanwoo has been intensively selected based on carcass traits such as carcass weight, eye muscle area, and marbling (intramuscular fat) through progeny tests. Hanwoo progeny tests facilitated annual genetic gains in carcass traits that increased dramatically in Hanwoo populations (4), and may have affected genomic regions associated with these carcass traits in brown Hanwoo. Understanding the genetic mechanisms that lead to phenotypic changes requires identification of the genomic regions that have been under long-term artificial selection.

Strong artificial selection increases the frequency of favorable alleles at the loci that affect meat quality traits in meat production breeds. In this process, a small region of the genome surrounding the mutations is also selected, resulting in a small genomic region that shows reduced variation. This region of reduced variation is referred to as a selection signature, which is identified by nucleotide distribution around favorable mutations that statistically differ from those expected purely by chance (5). By identifying these signatures of past selection and then identifying the functional genes and mutations involved, it is possible to identify the major genetic and metabolic pathways that control important agricultural characteristics of modern breeds. Searches for selection signatures have successfully revealed many genes that have been important in cattle selection. Qanbari et al. performed a whole genome scan for selection signatures in the Holstein genome and then detected a set of 10 candidate regions, including diacylglycerol O-acyltransferase 1, casein cluster, growth hormone receptor, somatostatin, and leptin receptor (6), based on extended haplotype homozygosities (EHHs). More recently, based on EHHs, Schwarzenbacher et al. (2012) demonstrated a combination of association analyses and selection signatures in dairy cattle (7); Gautier et al. (2011) looked for footprints of selection within a creole cattle breed (8); MacEachern et al. reported the results of a comparison of allelic frequencies between Australian Angus and Holstein cattle (9); Flori et al. (2009) (10) and Hayes et al. (2009) (11) also reported that the growth hormone recepeor (GHR) gene on Bos taurus autosome (BTA) 20 has selection evidence by comparing Holstein breeds, Angus and Holstein; and Gautier

ISSN: 1976-670X (electronic edition)

Copyright (C) 2016 by the The Korean Society for Biochemistry and Molecular Biology

(c) This is an open-access article distributed under the terms of the Creative Commons Attribution Non-Commercial License (http://creativecommons.org/licenses/by-nc/4.0) which permits unrestricted non-commercial use, distribution, and reproduction in any medium, provided the original work is properly cited. 
et al. (2009) performed a whole genome Bayesian scan in West African cattle (12). The aim of this study is to compare EHHs of brown and brindle Hanwoo. The candidate selection regions might have been shaped by intensive artificial selection in the brown Hanwoo, and therefore could contain the genes that affect the traits emphasized in the Korean breeding program.

\section{RESULTS AND DISCUSSION}

\section{Population structure and diversity}

PCA separated and grouped individuals based on their origins (Supplementary data 1). The 10 breeds (Brahman, five European, and four Northeast Asian breeds) formed tight clusters, which is evidence of high relatedness based on the genetic background of individuals that belong to the same breed. The distribution of animals in the first and second principal component $(\mathrm{PC} 1 \times \mathrm{PC} 2)$ was similar to the distribution observed in previous studies (Porto-Neto et al. 2013). The Hanwoo cattle clustered tightly with the Northeast Asian samples (e.g., Jeju black, Brindle, and Yanbian), whereas the Yanbian cattle (from the Chinese Yanbian region) clustered closer to the European samples by approximately one-third of the distance along the PC2 vector.

\section{Selection signal identification in brown Hanwoo}

The EHH approach is useful when we consider brindle Hanwoo as an ancestral breed to brown Hanwoo: recent selection in brown Hanwoo yielded divergence from brindle Hanwoo, which should be reflected by genetic analysis. Fig. 1 shows the iES plot for positively selected regions identified by our genome-wide scan. From a total of 37,770 single nucleotide polymorphisms (SNPs), we removed 74 SNPs as outliers and then searched for selection signals in brown Hanwoo. A total

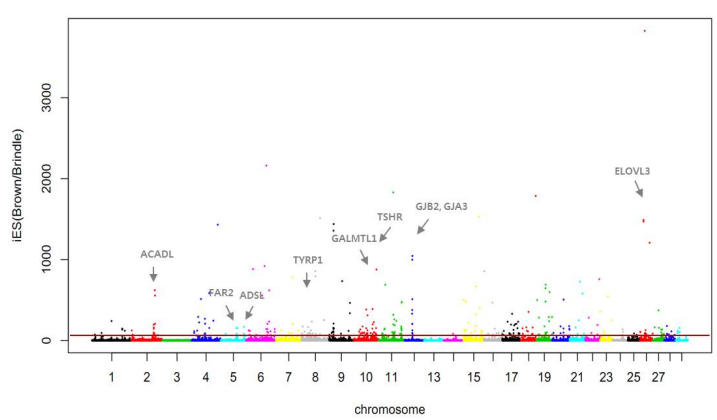

Fig. 1. Genome-wide map of $\mathrm{EHH}_{i}=\mathrm{iES}_{i, B \text { rown }} / \mathrm{iES}_{i, B r i n d l e}$. The $\mathrm{x}$-axis indicates chromosome position (Mb). The $y$-axis is the standardized transformed $\mathrm{z}$ value. We described the genes in the candidate regions that have been reported for meat traits.

Table 1. Genomic regions with significant evidence for relatively recent selection in brown Hanwoo comparing brindle Hanwoo

\begin{tabular}{|c|c|c|c|}
\hline $\begin{array}{l}\text { Chromosome } \\
\text { interval }(\mathrm{Mb})\end{array}$ & $\begin{array}{c}\text { Number of } \\
\text { significant SNPs }\end{array}$ & Genes & Gene name or Functional Annotation \\
\hline $1: 128.32-128.70$ & 5 & PCOLCE2, PSL1 & - \\
\hline $2: 102.88-103.52$ & 4 & UNC80, RPE, CPS1, ACADL & Fatty acid metabolism (22) \\
\hline 5:86.3-.87.7 & 4 & FAR2, CCDC91 & Lipid biosynthetic process \\
\hline 5:118.52-118.89 & 3 & TNRC6B, ADSL, SGSM3 & Muscle development, ATP metabolism \\
\hline 6:92.84-93.37 & 4 & PARM1 & - \\
\hline 9:19.65-20.54 & 8 & SH3BGRL2, TTK, BCKDH8 & - \\
\hline $10: 45.75-45.85$ & 5 & No genes found & - \\
\hline 10:83.44-84.24 & 4 & GALNTL1, SLC39A9, PLEKHD1, SRSF5, SMOC1 & Intramuscular adipocyte differentiation (23) \\
\hline $10: 94.45-96.35$ & 5 & TSHR & $\begin{array}{l}\text { Thyroid-stimulating hormone receptor, } \\
\text { influence carcass trait }\end{array}$ \\
\hline $11: 58.48-59.31$ & 5 & REG3G & - \\
\hline 11:95.21-96.69 & 6 & DAB2IP, MORN5, MRRF & - \\
\hline $12: 35.90-36.87$ & 8 & $\begin{array}{l}\text { ADHHC20, SKA3, XPO4, N6AMT2, GJB2, GJA3, } \\
\text { ZMYM5, PSPC1 }\end{array}$ & $\begin{array}{l}\text { Gap junction channel activity, } \\
\text { cell communication }\end{array}$ \\
\hline $15: 74.58-74.94$ & 3 & CD82 & Immune response \\
\hline $20: 51.22-51.80$ & 3 & $\mathrm{CDH} 10$ & - \\
\hline $26: 22.24-23.96$ & 3 & $\begin{array}{l}\text { TLX1, BTRC, DPCD, PPRC1, NOLC1, ELOVL3, HPS6, } \\
\text { GBF1, NFKB2, FBXL15, TMEM180, SUFU, TRIM8, } \\
\text { AS3MT, CNNM2 }\end{array}$ & $\begin{array}{l}\text { Elongation of long-chain fatty acids, } \\
\text { brown adipocyte differentiation (24), } \\
\text { muscle development (25) }\end{array}$ \\
\hline $28: 10.78-11.42$ & 3 & CHARM3 & - \\
\hline 29:18.41-20.18 & 4 & NARS2, ALG8, KCTD21, NDUFC2, INTS4, CLNS1A, PAK1 & - \\
\hline
\end{tabular}

The Genes column list annotated genes within the corresponding regions. Candidate genes in each region are indicated by bold type. 
of 306 SNPs were identified as having significantly different $\mathrm{EHH}$ values between the two populations. Of these, we deduced 17 core regions (more than three SNPs per region) in both directions up to $1.5 \mathrm{Mb}$ from the most significant SNP and annotated a subset of genes in the core region. Table 1 shows the core regions among candidate regions with strong signals in brown Hanwoo. We also found the overlapping region in the Animal quantitative trait loci $(\mathrm{QTL})$ database (http://www.animalgenome.org/cgi-bin/QTLdb/BT/index) (Supplementary data 2). The Animal QTL database revealed the presence of QTLs for meat traits (longissimus muscle area) and production traits (pre-weaning average daily gain) in cattle that overlapped with our candidate regions on chromosomes 5 (118.1 to $119.1 \mathrm{Mb}$ ) and 12 (36.1 to $37.1 \mathrm{Mb}$ ).

\section{Resequencing of candidate regions}

To further investigate the observed selection signatures, we selected four putative candidate regions among the selection signatures for brown Hanwoo from the results of $\mathrm{IES}$ analysis using the bovine SNP chip. First, we selected the core region (BTA12: 36.1-37 M) that was the most significant SNP in the candidate regions. We also selected the core regions (BTA5: 118.1-119.1 Mb and 86.3-87.8 Mb) that included the candidate genes (adenylosuccinatelyase, ADSL; and fatty acyl-CoA reductase 2, FAR2) for meat quality traits, as previously reported $(13,14)$. Finally, the candidate region (BTA14: 34.1-35.6 Mb) was selected, although it only had one significant SNP. However, BTA14 is well known to play a critical role that affects meat traits and thus will provide bare information from next-generation sequencing (NGS) data $(15,16)$. The detailed gene list is provided in Supplementary data 3.

Sequence data analysis showed a total of 250,800,964 sequence reads across the bovine genome. Of these, $196,378,008$ reads $(78.3 \%)$ were mapped to the bovine genome. The target enrichment was designed for selection signals totaling $4.86 \mathrm{Mb}$, and $55 \%$ of mapped reads were

Table 2. Homozygosity of SNPs in target genome

\begin{tabular}{lcc}
\hline \multicolumn{1}{c}{ Breed type } & $\begin{array}{c}\text { Brown } \\
\text { Hanwoo }\end{array}$ & $\begin{array}{c}\text { Brindle } \\
\text { Hanwoo }\end{array}$ \\
\hline Overall SNPs $(0.2 \leq$ freq) & 9,134 & 7,426 \\
Homo SNPs $(0.9 \leq$ freq) & 2,294 & 1,328 \\
Floating SNPs (0.2 $\leq$ freq $<0.9)$ & 6,840 & 6,098 \\
Homo SNP fraction (\%) & 25.1 & 21.7 \\
Homozygosity (mean \pm SD)* & $0.99 \pm 0.025$ & $0.96 \pm 0.04$ \\
\hline
\end{tabular}

Overall SNPs $(0.2 \leq$ frequency) were identified using vcftoolsand were classified into homo SNPs $(0.9 \leq$ frequency) and floating SNPs $(0.2 \leq$ frequency $\leq 0.9)$. *indicates significant difference of mean homozygosity between brown and brindle Hanwoo (Welch t-test, $\mathrm{P}$ value $=2.8089 \mathrm{E}-92$ ). The homozygosity is a mean value of allele frequency of homo SNPs. mapped to the target region for enrichment efficiency. Supplementary data 4 shows the summary statistics of sequence generation and mapping to the reference genome. The average depth over all samples was approximately $120 \times$. On average, $\sim 50 \%$ of all targets covered a depth of greater than $120 \times$, except for one sample (brown 4). We also measured mean homozygosity (mean frequency of each SNP) in the brown and brindle Hanwoo genomes (Table 2). The mean homozygosity of the brown Hanwoo genome was slightly higher than that of the brindle Hanwoo genome (0.99 versus 0.96). Many SNPs might have been fixed in the current brown Hanwoo genome during its history. In contrast, many genomic regions might still be under genetic drift for brindle Hanwoo. This supports the conclusion that brown Hanwoo have had many genomic regions selected during their history.

\section{Candidate gene mutation effects}

We performed SNP annotation based on the functional type in the target region. We identified a total of 10,314 SNPs in the candidate regions. Among 32 genes, functional impact of 29 nonsynonymous SNPs (nsSNPs) were analyzed using PolyPhen (Supplementary data 5), which predicts if the SNP affects the phenotype of the protein encoded by the gene carrying the SNP. PolyPhen analysis revealed 10 damaging nsSNPs and 19 benign nsSNPs. Interestingly, three nsSNPs (Lys88Met, Leu189His, and Arg302GIn) in the ADSL gene have the potential to have produced alterations in protein structure or function. Recently, ADSL was determined to be significantly expressed in longissimus muscle of high-fat groups of pigs that were $100 \mathrm{Kg}$ in body weight (13). Additionally, an ADSL polymorphism is associated with meat quality in chickens because it affects inosine 5'-monophosphate contents (14). Therefore, we focused on the effect of three ADSL nsSNPs and conducted a study to predict conformational changes of single residue substitutions and their impact on enzyme stability and ligand-binding affinity in the bovine ADSL/reaction products interaction system, which consists of only monomers, from the wild-type complex model. Separate subunit of the whole interaction system as a tetramer might found not to be equally effective of residue mutations as result in Table 3. Three ADSL variants showed differences between the folding free energy of mutated structures and binding free energy with those molecular partners in the mutated complex structures. The substitutions were not directly changed in binding affinity by the two ADSL products. However, they transmuted enzyme stability, which might affect the formation of the stabilizing tetramer directly related with its activity. Interestingly, the destabilizing effect of two mutations (L189H, R302Q) displayed relatively smaller structural changes in the monomeric than dimeric enzyme, which is conjugated with the stability of the active cleft by immediately adjacent variants. If the destabilizing extent disproportionality varies from monomeric to dimeric enzymes, it may reflect differences in how its tetramer nature and three subunits that form each active site 
Table 3. Evaluating effect of single-point mutations on stability and binding affinity in the dimer enzyme bound on reaction products

\begin{tabular}{|c|c|c|c|c|c|c|}
\hline & $\begin{array}{c}\text { Monomer } \\
\text { bovine ADSL }\end{array}$ & $\begin{array}{l}\text { Monomer } \\
\text { bovine ADSL }\end{array}$ & $\begin{array}{c}\text { Monomer } \\
\text { bovine ADSL }\end{array}$ & $\begin{array}{c}\text { Dimer } \\
\text { bovine ADSL }\end{array}$ & $\begin{array}{c}\text { Dimer } \\
\text { bovine ADSL }\end{array}$ & $\begin{array}{c}\text { Dimer } \\
\text { bovine ADSL }\end{array}$ \\
\hline Mutation & Lys88Met & Leu189His & Arg302Gln & L88Met & Leu189His & Arg302Gln \\
\hline Mutation Energy (stability) $^{\dagger}$ & $-0.42 \mathrm{kcal} / \mathrm{mol}$ & $1.28 \mathrm{kcal} / \mathrm{mol}$ & $3.45 \mathrm{kcal} / \mathrm{mol}$ & $-0.17 \mathrm{kcal} / \mathrm{mol}$ & $1.41 \mathrm{kcal} / \mathrm{mol}$ & $3.89 \mathrm{kcal} / \mathrm{mol}$ \\
\hline Stability effect* & Neutral & Destabilizing & Destabilizing & Neutral & Destabilizing & Destabilizing \\
\hline Mutation Energy (AMP binding) ${ }^{\ddagger}$ & $0.00 \mathrm{kcal} / \mathrm{mol}$ & $0.01 \mathrm{kcal} / \mathrm{mol}$ & $0.01 \mathrm{kcal} / \mathrm{mol}$ & $-0.03 \mathrm{kcal} / \mathrm{mol}$ & $-0.01 \mathrm{kcal} / \mathrm{mol}$ & $-0.01 \mathrm{kcal} / \mathrm{mol}$ \\
\hline AMP binding effect* & Neutral & Neutral & Neutral & Natural & Neutral & Neutral \\
\hline
\end{tabular}

${ }^{\dagger}$ The structure stability of themselves is evaluated as the differences between the folding free energy of mutated structures and the wild-type enzyme for the single-point mutations. ${ }^{\ddagger}$ The binding free energy is defined as the difference between the free energy of the complex and unbound state. *Mutation effect is defined as follows that stabilizing is indicate mutation energy is less than $-0.5 \mathrm{kcal} / \mathrm{mol}$ and Neutral is that mutation energy is between -0.5 to $0.5 \mathrm{kcal} / \mathrm{mol}$.

are affected by the mutations. Thus, it might produce a nonparallel reduction in the enzyme's catalytic activity. The decreasing stability of bovine ADSL by L189H and R302Q mutations in the dimeric form could also be regulated by multiple effects between the subunits to remove any unfavorable contacts that occurred within their neighboring interfaces. We described the detailed information in Supplementary data 6 .

Over the past 30 years, the body weight at 18 months of age increased from 331 to $574 \mathrm{~kg}$. The annual genetic gain also increased from 0.02 to $0.82 \mathrm{~kg} /$ year. Consequently, it was assumed that Hanwoo cattle might have dramatically increased genetic improvement. These results indicate that, although brown Hanwoo have experienced recent selective pressure with short divergence time, selection signatures have been observed with a fitness advantage during domestication. In this study, we compared EHHs between brown and brindle Hanwoo populations. We identified four candidate regions that may have been under recent selection in brown Hanwoo. These regions contained candidate genes associated with meat traits. We subsequently resequenced the candidate regions and focused on the ADSL gene, which was previously reported to be associated with meat quality. Three nsSNPs of this gene that affected the protein structure were identified though sequencing and protein structure modeling.

\section{MATERIALS AND METHODS}

\section{Animals and genotype assays}

Hanwoo data were collected from 140 steers in two populations of brown and brindle Hanwoo: a) steers of candidate bulls for progeny testing in the Hanwoo Improvement Center of National Agricultural Cooperative Federation in Seosan ( $\mathrm{n}=120)$; and b) steers raised in Hankyoung Univ. and Gyeonbuk Province $(n=20)$. Genomic DNA for genotyping assays was extracted from a blood sample, and SNP genotyping was performed using the Illumina Bovine SNP
50K Bead chip (Illumina, San Diego, USA). The protocol was approved by the Committee on the Ethics of Animal Experiments of the National Institute of Animal Science.

\section{SNP statistics analysis}

Stringent filtering criteria were applied to the genotype data. Briefly, SNPs were excluded from the analysis if they were absent from over $5 \%$ of the genotypes, had median GC scores below 0.6 , had GC scores under 0.6 in less than $90 \%$ of the samples, deviated in heterozygosity more than three standard deviations from the other SNPs, and were out of HardyWeinberg equilibrium (HWE) at a cutoff $\mathrm{P}$ value of $1^{-15}$. Unmapped SNPs and SNPs on sex chromosomes were also excluded. Individual genotypes with GC scores under 0.6 were treated as absent. After this - SNP filtering, genotypes were tested for HWE to identify possible typing errors using a chi-square test in R/SNPassoc Package ( $R$ Development Core Team). SNPs not in HWE (P<0.05), monomorphic SNPs, and those with a minor allele frequency $<1 \%$ were removed from this study. Finally, from a total of 55,704 SNPs, genotype data were received for 39,404 SNPs, and all of those SNPs were physically mapped to a chromosome (in bp) using the bovine genome sequence (Btau4.2).

\section{Hanwoo population structure analyses}

To characterize population structure for Hanwoo cattle, fixation indices $\left(F_{S T}\right)$ were estimated using the method described by Weir and Cockerham (1984) between East Asian ( $n=5$ breeds), European taurine breeds ( $n=4$ breeds), and an indicine breed (Brahman) with a reduced dataset of 20 randomly selected animals from each breed. Results were inferred based on a symmetrical distance matrix for the unrooted neighbor-joining tree estimation using R/APE package (Paradis et al, 2004). The genotype relationship matrix was estimated using VanRaden's algorithm (VanRaden, 2007) to infer similarities within and between breeds for principal component analyses. 


\section{Extended Haplotype Homozygosity (EHH)}

The counting algorithm of Tang et al (2007) was implemented to identify differential EHH regions within brown Hanwoo compared with brindle Hanwoo. To infer the proportion of homozygous individuals, $\mathrm{EHHS}_{i, j}$, at the $i$ th and $j$ th SNP were calculated using two steps. First, for each $\mathrm{SNP}_{i}, \mathrm{EHHS}_{i, j}$ between $\mathrm{SNP}_{i}$ and incrementally distant flanking $\mathrm{SNP}_{j}$ were calculated until $\mathrm{EHHS}_{i}, k<0$; this was performed for both $j>i$ and $j<\mathrm{I}$.

$$
E H H S_{(\text {geno }), j}=\frac{\sum_{k=1}^{n} I_{k,(\text { hap } i j)}\left(1 \text { if hap }_{1}=\text { hap }_{2}\right)}{\sum_{l=1}^{n} I_{l,(\text { alle } i)}\left(1 \text { if alle }_{1}=\text { alle }_{2}\right)}
$$

Second, the EHH of SNP $i$ was calculated: $\mathrm{iES}_{i}=\left(\mathrm{EHHS}_{i, j}\right)$ for $i j_{-} k$ for the 3 ' region of $I$ (or $i{ }_{j}{ }_{k} k$ for the 5 ' region of $i$ ).

$$
i E S_{i}=\sum_{j=a+1}^{b} \frac{\left(E H H S_{i, j-1}+E H H S_{i, j}\right)\left(\operatorname{Pos}_{j}-\operatorname{Pos}_{j-1}\right)}{2}
$$

Differential regions of $\mathrm{EHH}$ between brown and brindle Hanwoo were plotted based on the standardized of $\mathrm{iES}_{i}$ ratio between the two populations and compared with the EHHS decay of a single site. We calculated a standardized integrated EHH SNP [iES(z)] value to identify significant regions of positive selection ( $P=0.001, z=3.5)(17)$. We also discarded top and bottom $0.1 \%$ as outliers for incomplete signals across genomes. The remaining SNPs were defined as test SNPs. Candidate regions with positive selection footprints were defined as containing at least three SNPs $(P<0.001)$ with a $1.5-\mathrm{Mb}$ window and $0.75-\mathrm{Mb}$ overlap. If there were several continuous windows with three SNPs or more, we combined all overlapping windows into one candidate region using the intersectBed algorithm in BedTools (http://bedtools.readthedocs. io/en/latest/).

\section{Targeted resequencing of candidate regions}

We generated NGS data from two Hanwoo breeds including brown $(n=10)$ and brindle $(n=10)$ Hanwoo. We randomly sheared $3 \mu \mathrm{g}$ of genomic DNA using the Covaris System to generate $\sim 150 \mathrm{bp}$ inserts. The fragmented DNA was endrepaired using T4 DNA polymerase and Klenow polymerase, and Illumina paired-end adaptor oligonucleotides were ligated to the sticky ends. We analyzed the ligation mixture by electrophoresis on an agarose gel, and sliced and purified 200-250 bp fragments. The purified DNA library was hybridized with a SureSelect custom enrichment probes set (Agilent, Wilmington, USA) to capture a $4.86 \mathrm{Mb}$ targeted region following the manufacturer's instructions. We sequenced the Genome Analyzer IIx paired-end flowcell based on the manufacturer's protocol using captured exome library.

\section{Detection of variants and mutation effects of nsSNPs}

The sequences were aligned to the bovine reference genomes
(Btau 4.2) using the Burrows-Wheeler Aligner (version 0.6.1) (18) with default parameters. SAMtools (version 0.1.17) (19) was used to convert (SAM/BAM), sort, and index alignments. We used Picard tools to generate quality matrices for mapping. Duplicated reads were marked with Picard tools and excluded from downstream analysis. We performed local re-alignment and re-calibration using the Genome Analysis Toolkit (version 1.5.9) framework (20). We also used variant calling to perform variant calls and extract corresponding quality scores for each genotype. Raw SNP calls were filtered using empirically derived cut-offs for the following GATK filter expressions: clusterSize 3, - clusterWindowSize 10,- maskNamelndelfilterExpression "MQ $>=4$ \&\& ((MQO/(1.0*DP)) $>0.1)^{\prime \prime-}$ filterExpression "QUAL $<30$ ", -- filterExpression "QD $<5.0$ "filterExpression "Hrun $>$ 5", -filterExpression "FS $>200.0$ ". We performed SNP annotation based on functional type using ANNOVAR (21) and filtered against known variants by comparison to the NCBI dbSNP database. nsSNPs were detected and the SNP/gene functional property analyzed using PolyPhen (http://genetics.bwh.harvard.edu/pph2). To explore mutation effects of candidate genes, we predicted the bovine protein structure by comparative homology modeling with the only of template structure (PDB code 2VD6) as human protein for which structure is adapted in and around its active sites of ligand binding with the way that subunits are assembled into dimers and tetramers. The homology modeling was performed using MODELER9 (version 10) within Discovery Studio (version 3.5) molecular modeling packages (http://accelrys.com/ products/discovery-studio/; Accelrys Inc, San Diego, USA).

\section{ACKNOWLEDGEMENTS}

This work was supported by Agenda (PJ01040601 and PJ90695602) of the National Institute of Animal Science, Rural Development Administration (RDA), Republic of Korea.

\section{REFERENCES}

1. Seo K, Mohanty TR, Choi T and Hwang I (2007) Biology of epidermal and hair pigmentation in cattle: a minireview. Vet Dermatol 18, 392-400

2. Choi TJ (2009) establishment of phylogenetic characteristics for korean traditional cattle breeds. Chonbuk National University.

3. Yoon D, Kwon Y, Lee K et al (2008) Discrimination of Korean Cattle (Hanwoo) Using DNA Markers Derived from SNPs in Bovine Mitochondrial and SRY Genes. Asian-Australas J Anim Sci 21, 25-28

4. Kim H, Lee S, Cho Y et al (2011) Genomic Information and Its Application in Hanwoo (Korean Native Cattle) Breeding Program - A Mini Review. Ann Anim Resour Sci 22, 125-133

5. Kim Y and Stephan W (2002) Detecting a local signature of genetic hitchhiking along a recombining chromosome. Genetics 160, 765-777 
6. Qanbari S, Pimentel EC, Tetens J et al (2010) The pattern of linkage disequilibrium in German Holstein cattle. Anim Genet 41, 346-356

7. Schwarzenbacher $H$, Dolezal $M$, Flisikowski $K$ et al (2012) Combining evidence of selection with association analysis increases power to detect regions influencing complex traits in dairy cattle. BMC Genomics 13, 48

8. Gautier M and Naves M (2011) Footprints of selection in the ancestral admixture of a New World Creole cattle breed. Mol Ecol 20, 3128-3143

9. MacEachern S, Hayes B, McEwan J and Goddard M (2009) An examination of positive selection and changing effective population size in Angus and Holstein cattle populations (Bos taurus) using a high density SNP genotyping platform and the contribution of ancient polymorphism to genomic diversity in Domestic cattle. BMC Genomics 10, 181

10. Flori L, Fritz $\mathrm{S}$, Jaffrezic $F$ et al (2009) The genome response to artificial selection: a case study in dairy cattle. PLoS One 4, e6595

11. Hayes B, Chamberlain A, Maceachern S et al (2009) A genome map of divergent artificial selection between Bos taurus dairy cattle and Bos taurus beef cattle. Animal Genetics 40, 176-184

12. Gautier M, Flori L, Riebler A et al (2009) A whole genome Bayesian scan for adaptive genetic divergence in West African cattle. BMC Genomics 10, 550

13. Liu J, Damon M, Guitton N et al (2009) Differentiallyexpressed genes in pig Longissimus muscles with contrasting levels of fat, as identified by combined transcriptomic, reverse transcription PCR, and proteomic analyses. J Agric Food Chem 57, 3808-3817

14. Ye M, Chen J, Zhao G, Zheng M and Wen J (2010) Correlation between polymorphisms in ADSL and GARSAIRS-GART genes with inosine 5'-monophosphate (IMP) contents in Beijing-you chickens. Br Poult Sic 51, 609-613

15. Lee SH, Choi BH, Lim D et al (2013) Genome-wide association study identifies major loci for carcass weight on BTA14 in Hanwoo (Korean Cattle). PLoS One 8, e74677

16. Lee S, Van Der Werf J, Park E, Oh S, Gibson J and Thompson J (2010) Genetic polymorphisms of the bovine fatty acid binding protein 4 gene are significantly associated with marbling and carcass weight in Hanwoo (Korean Cattle). Animal Genetics 41, 442-444

17. Tang K, Thornton KR and Stoneking M (2007) A new approach for using genome scans to detect recent positive selection in the human genome. PLoS Biol 5, e171

18. Li H and Durbin R (2009) Fast and accurate short read alignment with Burrows-Wheeler transform. Bioinformatics $25,1754-1760$

19. Li H, Handsaker B, Wysoker A et al (2009) The Sequence Alignment/Map format and SAMtools. Bioinformatics 25, 2078-2079

20. McKenna A, Hanna M, Banks E et al (2010) The Genome Analysis Toolkit: a MapReduce framework for analyzing next-generation DNA sequencing data. Genome Res 20, 1297-1303

21. Wang K, Li M and Hakonarson H (2010) ANNOVAR: functional annotation of genetic variants from highthroughput sequencing data. Nucleic Acids Res 38, e164

22. Taniguchi M, Guan LL, Basarab JA, Dodson MV and Moore SS (2008) Comparative analysis on gene expression profiles in cattle subcutaneous fat tissues. Comp Biochem Physiol Part D Genomics Proteomics 3, 251-256

23. Mizoguchi Y, Hirano T, Itoh T et al (2010) Differentially expressed genes during bovine intramuscular adipocyte differentiation profiled by serial analysis of gene expression. Anim Genet 41, 436-441

24. Jorgensen JA, Zadravec D and Jacobsson A (2007) Norepinephrine and rosiglitazone synergistically induce Elovl3 expression in brown adipocytes. Am J Physiol Endocrinol Metab 293, E1159-1168

25. Reverter A, Hudson NJ, Wang Y et al (2006) A gene coexpression network for bovine skeletal muscle inferred from microarray data. Physiol Genomics 28, 76-83 